\title{
The Connectedness Between Society Response and the Stress of Adolescents with Non-Suicidal Self-Harm Behavior
}

\author{
Ximin $\mathrm{Bao}^{1, \dagger}$, Yayu $\mathrm{Sun}^{2, *}{ }^{*} \dagger$ and Shenyi $\mathrm{Wu}^{3, \dagger}$ \\ ${ }^{1}$ Beijing New Oriental Foreign Languages School, Yangzhou, Jiangsu, China \\ ${ }^{2}$ Shenzhen Vanke Meisha Academy, Shenzhen, Guangdong, China \\ ${ }^{3}$ The Affiliated International School of Shenzhen University, Shenzhen, Guangdong, China \\ *Corresponding author.Email: sunyayu@stu.vma.edu.cn \\ Those authors contributed equally.
}

\begin{abstract}
This paper reviews existing studies relevant to adolescents' non-suicidal self-harm behavior. This paper summarizes, interprets, and analyzes these research outcomes in the aspects of internal and external causes, stigmatization from society and its consequences, and prevention and intervention, which lead to the conclusion that non-suicidal self-harm is a serious and urgent problem worth paying great attention to. Meanwhile, it raises new questions and offers potential solutions and research directions in order to comprehensively enrich the studies of the overall process of adolescents' self-harm behaviors comprehensively. This paper provides researchers with more specific ideas focusing on adolescents experiences of stigma and further mental health issues.
\end{abstract}

Keywords: non-suicidal self-harm, adolescents, social stigma, mental well-being.

\section{INTRODUCTION}

\subsection{Research Background}

Self-harm includes acts such as cutting, punching, and burning with cigarettes, matches, and candles, all of which are intended to cause harm to oneself. Adolescents whom self-harm may experience mental health issues to some level because it is a negative way of expressing and dealing with important passive emotions. Self-harm and suicide are important public health concerns among adolescents, with rates of self-harm rising during adolescence and suicide being the second largest cause of death among teenagers worldwide [1]. Self-harm among teenagers is clearly a problem, with community surveys revealing that 3-10\%of adolescents have experienced at least one incident of self-harm in the previous year, with lifetime rates of 9-14\% [2]. About half of all teenagers who self-harm will do so more than once; cutting and overdosing are the most common ways, and only a small percentage of self-harming episodes will result in hospitalization. An additional $15-22 \%$ of young people said they had thoughts of self-harm in the previous year but didn't act on them [3]. Hankin and Abela studied the effects of self-harm on 103 young adolescents aged 11 to 14. Over the course of the study's 212 years, 18 percent of early adolescents self-harmed, with 14 percent of those cases being new. Negative cognitive style, depressed symptoms, and a lack of social support were all linked to self-harm. While half of the individuals who self-harmed at baseline continued to self-harm at follow-up, no factors linked with cessation of continuing could be investigated [4]. Self-harm without suicidal intent was found to be $3 \%$ of the time. Self-harmers were more likely to be female, to have depressive symptoms and somatic issues, to smoke cigarettes, and to know someone who had selfharmed. Over the course of two years, Lundh et al. surveyed a population sample of 1052 early adolescents aged 13 - 15 years in Sweden twice. Someone who has caused harm to themselves. Over the course of two years, Lundh et al. surveyed a population sample of 1052 early adolescents aged 13 - 15 years in Sweden twice [5]. The causes of self-harm and the need of preventing NSSI have also been discussed in prior studies. There is a large body of literature that outlines the risk factors for self-harm, which is important for developing interventions [6]. Age, ethnicity, family living circumstances, and family affluence all played a role in the development of self- 
harm. Only age and not going on vacation (an indicator of affluence) were related to self-harm after other sociodemographic variables were controlled for [7]. Self-harm is a serious problem that affects up to $25 \%$ of young people and can lead to negative consequences such as self-harm repeat, suicide and death, mental health morbidity, poorer education, and employment results, and overall lower quality of life, as well as being expensive to treat [8]. Mood problems and a history of abuse are also mentioned in community-based studies, as well as bullying as being more distant from self-harm, substance use, and psychological traits [9]. Currently, many therapies for young people who self-harm are clinic-based, administered by mental health specialists, and primarily oriented at treating the underlying mood and personality issues [10]. These interventions are vital, yet many young people do not seek out traditional faceto-face mental health services [11]. Moving on to the social stigmatization that exists. The term "stigma" refers to a lack of knowledge and prejudiced attitudes that lead to discriminatory behavior toward a group of individuals. a person's attitude toward a group of people 6 Public stigmas (prejudice held by the broader public toward those who self-harm) can be distinguished from selfstigma when it comes to self-harm (the negative attitudes that those who self-harm hold against themselves). 6 public stigma develops. 6 Such attributions are all too common, and those who self-harm frequently endorse negative preconceptions [12].

\subsection{Research Gap}

This paper specifically studies the plight of adolescents with social stigma caused by physical markers and necessary preventive measures. Differ from previous studies, this paper concentrates on the problems, summarizing and analyzing them with a broader scope than previous studies. Studies like medical and psychological research have focused on the single points - they were all single directional studies. However, this paper has a macro view. The objectives of this paper are to learn about more detailed and in-depth social problems through the writing process. In previous research, groups with self-harm behavior, the way they commit self-harm, and the significance of avoiding selfharm are common contents. However, few studies have specifically written about the psychological changes and a series of the aftermath of people committing self-harm like whether their emotions become positive or negative and whether they gain excitement or a sense of release from it. Subsequent questions include how the frequency of self-harm fluctuates, what are the fundamental reasons behind these fluctuations, how the society treats these minorities, the reasons for discrimination, and its approaches. These processes further infer methods the society should adopt to treat these minorities correctly and fairly.

\section{CAUSES OF NON-SUICIDAL SELF- HARM BEHAVIOR}

\subsection{Mental illness}

One of the most common causes for teens to selfharm is mental health issues. Some people suffering from depression or anguish may begin self-harming in order to relieve excruciating mental agony or gain control over their lives. The issue is that the alleviation they experienced initially may wane over time. Individuals may become more frequent or intense in their selfharming behavior as a result of this, putting their lives in jeopardy, for example, by accidentally overdosing. Selfharming can lead to suicidal conduct in some people. Self-harm is frequently considered to arise as a sign of severe mental discomfort (anxiety, depression, self-hate, etc.) and as a means of expressing or managing this distress [13]. This would imply that negative emotions (such as depression) are a risk factor for the development of self-harm. A risk factor is a measurable characteristic that must occur before a negative event and be linked to a higher chance of that outcome, which implies risk factors can only be identified through prospective studies. There is a strong connectedness between mental problems, which is the cycle. They can gain temporary relief from self-harm, as well as emotion and feelings of guilt and shame, they would experience more serious mental health and a higher frequency of self-harm or NSSI. But mental health problems are not the only leading cause of juvenile self-harm behaviors, there are other factors, sexual minorities who are confused about their sexuality would also be a considerable cause to trigger the behavior of NSSI.

\subsection{Confusion of sexuality}

Lesbians, gays, bisexuals, and transgender persons can be proud of this. [14] From distal to proximal, external, objective stressful events and situations (chronic and acute), (b) expectations of such events and the vigilance demanded by such expectations, and (c) internalization of negative societal attitudes are enumerated. Another study, specifically psychological research in the field of disclosure, found that there is at least one more stressor to consider: concealing one's sexual orientation [14].

\subsection{Domestic relationship problems}

Domestic relationship problems act as an environmental factor in contributing to non-suicidal selfharm behaviors. As a crucial part of family relationships, the loss, break, or significant change in partnership can be a cause of self-harm. According to Althoff's study, the loss of a spouse affects adolescents' emotional state and mental health [15], thus increasing the risk of self-harm behaviors. Besides, healthy intimate relationships like 
parent-child relationships work similarly. Althoff's study also reveals that parents' behaviors function as a model or directly guide parents' attitude and treatment towards their children [15], and further impact adolescent's mental well-being. There is a clear relationship between parents' unhealthy and toxic life habits such as long-term abuse of drugs or alcohol and their children's non-suicidal self-harm behaviors [15] since parents' abuse behavior is a potential factor causing domestic abuse or maltreatment. Tao's study shows that bad and poor parent-child relationships can result in adolescents' nonsuicidal self-harm behaviors [16]. A lack of a proper parent-child relationship or a negative, toxic parent-child relationship causes adolescents to have no place to relieve their pressure, then self-harm will be a desperate way for them to express their negative emotions and release their stress. The absence of a trustable intimate relationship between adolescents and parents increases the risk of self-harm behavior.

\subsection{Domestic abuse or maltreatment}

As a type of extreme case, domestic violence or maltreatment from parents during childhood is proved to be a direct and significant factor of adolescents' nonsuicidal self-harm behaviors later in their adolescence. Based on Boyle's study, within the group of domestic violence victims, over $1 / 4$ of them show self-harm behavior after they encounter violent treatment from their parents [17]. A small group of victims also immediately commit self-harm after domestic violence. According to McMahon, besides domestic violence, simply maltreatment from adolescents' parents during childhood can increase the risk of self-harm behavior in the future [18]. His study reveals the strongly connected causal relationship between child maltreatment and violent behaviors toward adolescents themselves. In addition, adverse childhood experiences, as a child-oriented consequence of childhood maltreatment, have a positive correlation with non-suicidal self-harm behaviors. According to Carr's study, the potential causes of parents' maltreatment behaviors include their unemployment and the diagnosis of some mental disorders with symptoms of substance abuse [19]. These factors can lead to their violent treatment towards children and later trigger their self-harm behaviors. The childhood experiences of violence and maltreatment can be regarded as an indicator of the victims' self-harm behaviors in adolescence. Carr also reveals that the impact of adverse childhood experiences has a stronger effect compared with other factors like a loss of parents or a break of intimate relationships [19].

\subsection{Sexual abuse}

For adolescents, sexual abuse encountered in childhood plays a relevant role in leading to later nonsuicidal self-harms indirectly. Collin-Vézina's study reveals a positive relationship between child sexual abuse and self-injurious thoughts and behaviors later on. Victims of child sexual abuse imply the cause-and-effect relationship between their past experiences of sexual abuse and present unhealthy well-being and mental problems, which further explicitly show as invisible and visible self-harm symptoms [20]. The mental trauma resulting from child sexual abuse cause adolescent to commit self-harm behaviors after deteriorating their mental health and making them suffer from mental illness.

\section{STIGMATIZATION OF ADOLESCENTS WITH NON-SUICIDAL SELF-HARM BEHAVIOR}

\subsection{Stereotype}

Society has stereotypes about physical scars such as those from self-harm. There seems to be an inevitable correlation when some part of a person's body is exposed such as scars on the neck, wrists, arms, face[21]. People naturally associate these scars with something bad[21]. For example, it's assumed that these people are prone to substance abuse, personality disorders, and certain mental illnesses. Essentially negate this minority group. When a teenager has these scars in any setting. Often, parents of students will tell their children to stay away from this minority group. The ability to work in the workplace is not seen, and scars are often associated with incompetence. Extreme events occur in the environment, such as beatings, theft, etc. This minority group is subconsciously singled out as a suspect. Scars seem to be an ironic imprint on this minority group.

\subsection{Prejudice}

While personal expression appears to have some support in the legislative and law reform justifications of physical features, courts have been far less willing to lean on this justification. Instead, the case law appears to consider the need for a broad interpretation of the legislation within the definition of physical features but struggles to articulate a coherent justification for its interpretation. The conclusion is a confusing and, at times, contradictory body of case law. Even the law, it seems, is hard to balance. The length of the hair, the color of the hair, and the style of the earrings can legally be used as physical characteristics. Scarring does not seem to constitute a characteristic of a persuasive body. This means that minorities with scars and body markings who have been discriminated against have difficulty using reasonable means to rehabilitate themselves. It is not verbal and behavioral aggression that counts as discrimination. Some of the implied ways are not directly expressed is actually a kind of unfair treatment. 


\section{THE RELATIONSHIP BETWEEN PERPETRATION AND STIGMATIZED GROUP}

Stigma triggers status loss, or a drop in the social hierarchy. To the degree that this occurs, people of stigmatized groups are likely to experience a variety of negative consequences associated with reduced status, ranging from sexual partner selection to lifespan. Structural discrimination might have harmful consequences that have nothing to do with the stereotyped notions that inspired the discrimination in the first place. The Not In My Back Yard (NIMBY) phenomena, for example, resulted in mental health treatment facilities being located in relatively impoverished and powerless regions of the city, which were also crime-ridden and dangerous.[22]As a result, those with mental problems are far more vulnerable than others to be assaulted. Third, people's attempts to deal with stigma may have unintended repercussions that appear to have nothing to do with the stereotype [23]. Therefore, adolescents would be afraid to tell or share their emotions with others. They even have misunderstandings of their behavior and resulting from the internalized stigmatization. People experiencing or perceived to have a mental illness are often subject to negative stereotyping and biases (stigma) from many sources, producing multifaceted negative effects [24]. Internalized stigma has been associated with a number of negative outcomes, including increased depression, avoidant coping [25]; decreased hope and self-esteem, worsening psychiatric symptoms; and decreased persistence in accessing mental health services and other supports. Those results would cause more serious mental problems and a higher frequency of self-harm. so they would hide their negative emotions and behaviors rather than ask for help.

\section{CONSEQUENCES OF BEING STIGMATIZED}

\subsection{Suicide}

Suicide is a potential social influence. It includes acts that intentionally lead to suicide, also known as attempted suicide. It is a major public health problem. People who are prone to self-harm or suicide often suffer from serious mental illness. Often accompanied by personality disorders. Self-harm and suicide are often related to the surrounding living environment and acute life events. Such as relationship problems and financial difficulties or social isolation. Once self-harm or attempted suicide occurs, the risk of subsequent suicide increases significantly. At the same time, these behaviors are stigmatized by society. People will look at this minority group with colored glasses. Think that they have a bad history and will launch personal attacks. It will be the psychological defense of this minority group to break down again.

\subsection{More serious mental problem}

At least $80 \%$ of self-injurers have at least one mental disorder, including major depression, bipolar disorder, anxiety, and substance abuse, often with a personality disorder [26]. Patients with a history of self-harm and suicide who subsequently have severe psychological problems are at significantly increased risk of self-harm and suicide. Suicidal self-harm involves more than just complex psychological factors. Inincluding mental illness and social problems. It is also associated with poorer educational outcomes and huge health and social care [26]. Because of the high incidence of suicide and self-injury among teenagers, it is necessary to pay attention to their mental health and carry out a series of educational projects and preventive measures.

\subsection{Higher frequency of self-harm}

Repeated self-harm is common, with up to $25 \%$ of patients returning to the same hospital within a year of their initial cure. Repetition was also common among adolescents who did not participate in clinical services. People with a history of self-harm are high and capricious. Some teenagers self-harm after the release or pain and stimulation of some kind of pleasure, often when the mood fluctuations are a great direct use of selfharm to obtain pleasure and release. In the case of public opinion and emergency, they tend to have extreme rebellious psychology. And increased rates of self-harm. Due to the high frequency of self-injury among adolescents, improving the treatment of self-injury has become the focus of many national and international guidelines.

\section{SIGNIFICANCES OF PREVENTING NON-SUICIDAL SELF-HARM BEHAVIOR}

Self-harm behavior among young people has been studied extensively, with socioeconomic background, gender, and individual characteristics like sexual orientation and identity all being examined [30]. Females made up three-quarters of the participants $(7,150$ selfharm incidents). The female: male ratio was 5.0 in 10 14-year-olds, and 2.7 in 15-18-year-olds. The rates of self-harm did not differ significantly between the centers. Females had a rate of 302 per 100,000 (95 percent CI 269-335) in 10-14-year-olds and 1,423 (95 percent CI $1,346-1,501)$ in $15-18$-year-olds, while males had a rate of 67 (95 percent CI 52-82) and 466 (95 percent CI 422 510). Self-harm was a common occurrence (53.3 percent had a history of prior self-harm and 17.7 percent repeated within a year) (Hawton, K.2012). A history of nonsuicidal self-injury prior to trial enrollment was the only predictor of nonsuicidal self-injury (odds ratio $=9.6$ ). 
During the 36-week follow-up period of the Treatment for Adolescents with Depression Study (TADS) (11), $10 \%$ of participants reported suicidal episodes (half of which were suicide attempts). In univariate analysis, baseline suicidality and high levels of self-rated depressive symptoms predicted suicidal occurrences. Nonsuicidal self-injury did not have its own category [32]. Deliberate self-harm is a major problem for young people in modern times, the number of youngsters attending hospital after self-harm is increasing, and two large-scale community-based surveys of self-harm among young people in England have shown that rates of self-harm are much higher than reports of pure intention. More than 6,000 15-to 16-year-olds were polled in a representative sample of 41 schools in one study from Oxford's Centre for Suicide Research. Overall, 6.9\% (11.2 percent girls and 3.2 percent boys) reported an act of self-harm that fit the study's criteria in the previous year, with only 12.6 percent of these cases resulting in hospitalization. [33]. Wilkinson says it's critical to avoid self-harming or non-suicidal self-injury. Suicide risk did not differ over time between individuals who had suicidal and non-suicidal self-harm events at the start (2011). There're serious negative stereotypes on minority groups (adolescents with self-harm) like 'they are seeking attention or 'they are fragile in psychology'.

\section{INTERVENTION OF NON-SUICIDAL SELF-HARM BEHAVIOR}

Psychologists in the present have figured out the most efficient method for curing certain mental illnesses and preventing non-suicidal self-harm behaviors. Dialectical behavior therapy (DBT), also called dialectical behavioral therapy and cognitive-behavioral therapy (CBT), also called Cognitive-behavioral-based psychotherapy are the two commonest methods therapists use to deal with self-harm behavior. According to Ougrin's study, these two ways have the largest effect on the intervention of adolescents' self-harm behaviors [34]. He also mentions another type of therapy called mentalization-based therapy (MBT), which has a similar principle and effect on intervention compared with the other twos. Another researcher considers problemsolving therapy as part of CBT to be conducted together on adolescents who are committing self-harm [35]. His study reveals the prominent effect of problem-solving therapy on reducing the possibility of repeated self-harm behavior. Developmental group therapy, as another means, proved by Morken that is equally effective as DBT is [37]. Additionally, based on Turner's study, emotion regulation group therapy and manual-assisted cognitive therapy are also potential methods in preventing adolescents from committing self-harm behaviors. The essence and principle of all the therapies above are somehow the same. These therapies assist adolescents with non-suicidal self-harm behaviors to identify and regulate their emotions, change their thoughts about self-harm, and teach them proper ways of coping with issues. Therefore, more simple methods such as writing, playing or listening to relaxed and smooth music, painting, crocheting, and clay work [38] also mean that can help adolescents out in daily life since artistic expression are also good ways to control emotion and improve cognition.

For unprofessional people who intend to apply interventions to adolescents with non-suicidal self-harm behaviors such as their families and friends, several daily means works in the same way as professional therapies. Diversion techniques are effective in distracting adolescents' attention away from self-harm-relevant thoughts [38]. Though it's easily conducted, it's a typical avoidance behavior that cannot eliminate negative thoughts. Examples of diversion techniques include noncompetitive physical exercises that are vigorous enough for adolescents [38] such as jogging and walking and hiking, but these activities need to be carried out in proper time and safe places.

\section{CONCLUSION}

Non-suicidal self-harm, as a means for coping with negative emotion, has a relatively high rate within adolescent groups. Dozens of studies have presented the serious consequences of adolescents' self-harm behavior including mental health problems and social stigma. Therefore, reducing self-harm behavior and eliminate its resulting outcome is a crucial topic. There are two major categories of the causes of self-harm behavior; internal causes mental illness, which stimulate self-harm by exerting negative emotion and thoughts to the mentality of adolescents, and external causes include domestic relationship problem, domestic abuse or maltreatment, and sexual abuse, which unable adolescents to develop heathy and appropriate intimate relationship and lead to long-term psychological trauma. One of the Society's responses to adolescents' self-harm behavior is to stigmatize them with mainly stereotypes and prejudice.

A potential consequence of being stigmatized by the majority of society is suicide, since the perpetration of stigmatization further aggravates the existing mental illness or negative emotion, and then promotes suicide behavior, which is the extreme form of self-harm behavior. Research reveals that non-suicidal self-harm behaviors are predictable from adolescents' past experiences such as abuse and bullying, identity threat such as sexuality, and mental illness such as depression and anxiety. The lack of social support and regular therapy will finally lead to more serious and irreversible outcomes. Hence, it is possible and necessary to prevent self-harm behavior. The recent most effective psychological therapies for indirectly solving self-harm problems are dialectical behavior therapy and cognitivebehavioral therapy, which help adolescents develop proper self-recognition, regulate their emotion, and learn 
how to cope with issues properly. Attention-diversion, physical exercise, and artistic expression and creation are also feasible ways of preventing adolescents' nonsuicidal self-harm behaviors.

\section{REFERENCES}

[1] Hawton, K., Bergen, H., Waters, K., Ness, J., Cooper, J., Steeg, S., \& Kapur, N. (2012). Epidemiology and nature of self-harm in children and adolescents: Findings from The Multicentre study of self-harm in England. European Child \& Adolescent Psychiatry, 21(7), 369-377. https://doi.org/10.1007/s00787-012-0269-6

[2] Kokkevi, A., Rotsika, V., Arapaki, A., \& Richardson, C. (2011). Adolescents' self-reported suicide attempts, self-harm thoughts, and their correlates across 17 European countries. Journal of Child Psychology and Psychiatry, 53(4), 381 - 389. https://doi.org/10.1111/j.1469-7610.2011.02457.x

[3] Morey, C., Corcoran, P., Arensman, E., \& Perry, I. J. (2008). The prevalence of self-reported deliberate self harm in Irish adolescents. BMC Public Health, 8(1). https://doi.org/10.1186/1471-2458-8-79

[4] Hankin, B. L., \& Abela, J. R. Z. (2011). Nonsuicidal self-injury IN ADOLESCENCE: Prospective rates and risk factors in a 2 1/2year longitudinal study. Psychiatry Research, 186(1), 65-70. https://doi.org/10.1016/j.psychres.2010.07.056

[5] Lundh, L.-G., Wångby-Lundh, M., Paaske, M., Ingesson, S., \& Bjärehed, J. (2011). Depressive symptoms and deliberate self-harm in a community sample of adolescents: A prospective study. Depression Research and Treatment, 2011, 1-11. https://doi.org/10.1155/2011/935871

[6] Hetrick, S. E., Subasinghe, A., Anglin, K., Hart, L., Morgan, A., \& Robinson, J. (2020). Understanding the needs of young people who engage in self-harm: A qualitative investigation. Frontiers in Psychology, 10. https://doi.org/10.3389/fpsyg.2019.02916

[7] Stallard, P., Spears, M., Montgomery, A. A., Phillips, R., \& Sayal, K. (2013). Self-harm in young adolescents (12-16 years): Onset and short-term continuation in a community sample. BMC Psychiatry, 13(1). https://doi.org/10.1186/1471244x-13-328

[8] Fergusson, D. M., Boden, J. M., \& Horwood, L. J. (2008). Exposure to childhood sexual and physical abuse and adjustment in early adulthood. Child Abuse \& Neglect, 32(6), 607 - 619. https://doi.org/10.1016/j.chiabu.2006.12.018
[9] Plener, P. L., Schumacher, T. S., Munz, L. M., and Groschwitz, R. C. (2015). The longitudinal course of non-suicidal self-injury and deliberate self-harm: a systematic review of the literature. Borderline Personal. Disord. Emot. Regul. 2:2. doi: 10.1186/s40479-014-0024-3

[10] Hawton, K., Witt, K. G., Salisbury, T. L., Arensman, E., Gunnell, D., Hazell, P., Townsend, E., \& van Heeringen, K. (2016). Psychosocial interventions following self-harm in adults: A systematic review and meta-analysis. The Lancet Psychiatry, 3(8), 740750.https://doi.org/10.1016/s2215-0366(16)300700

[11] Whitlock, J., Eckenrode, J., and Silverman, D. (2006). Self-injurious behaviors in a college population. Pediatrics 117, 1939-1948. doi: 10.1542/peds.2005-2543

[12] Corrigan, P., Markowitz, F. E., Watson, A., Rowan, D., \& Kubiak, M. A. (2003). An attribution model of public discrimination towards persons with mental illness. Journal of Health and Social Behavior, 44(2), 162. https://doi.org/10.2307/1519806

[13] Chapman, A. L., Gratz, K. L., \&amp; Brown, M. Z. (2006). Solving the puzzle of deliberate self-harm: The experiential avoidance model. Behavior Research and Therapy, 44(3), 371-394. https://doi.org/10.1016/j.brat.2005.03.005

[14] Meyer, I. H. (2003). Prejudice, social stress, and mental health in lesbian, gay, and bisexual populations: Conceptual issues and research evidence. Psychological Bulletin, 129(5), 674-697. https://doi.org/10.1037/0033-2909.129.5.674

[15] Althoff, R. R., Hudziak, J. J., Willemsen, G., Hudziak, V., Bartels, M., \& Boomsma, D. I. (2012). Genetic and environmental contributions to selfreported thoughts of self-harm and suicide. American journal of medical genetics. Part B, Neuropsychiatric genetics: the official publication of the International Society of Psychiatric Genetics, $159 B(1)$,

$120-127$ https://doi.org/10.1002/ajmg.b.32010

[16] Tao, Y., Bi, X.-Y., \& Deng, M. (2020). The impact Of Parent-Child attachment On SELF-INJURY Behavior: Negative emotion and emotional Coping style as Serial mediators. Frontiers in Psychology, 11. https://doi.org/10.3389/fpsyg.2020.01477

[17] Boyle, A., Jones, P., \& Lloyd, S. (2006). The association between domestic violence and self harm in emergency medicine patients. Emergency 
medicine journal: EMJ, 23(8), 604-607. https://doi.org/10.1136/emj.2005.031260

[18] McMahon, K., Hoertel, N., Olfson, M., Wall, M., Wang, S., \& Blanco, C. (2018). Childhood maltreatment and impulsivity as predictors of interpersonal violence, self-injury and suicide attempts: A national study. Psychiatry research, 269, 386-393.

https://doi.org/10.1016/j.psychres.2018.08.059

[19] Carr, M. J., Steeg, S., Mok, P., Pedersen, C. B., Antonsen, S., Kapur, N., \& Webb, R. T. (2020). Adverse Childhood Experiences and Risk of Subsequently Engaging in Self-Harm and Violence towards Other People-"Dual Harm". International journal of environmental research and public health, 17(24), 9409. https://doi.org/10.3390/ijerph17249409

[20] Collin-Vézina, D., De La Sablonnière-Griffin, M., Sivagurunathan, M., Lateef, R., Alaggia, R., McElvaney, R., \& Simpson, M. (2021). "How many times did I not want to live a life because of him": the complex connections between child sexual abuse, disclosure, and self-injurious thoughts and behaviors. Borderline personality disorder and emotion dysregulation, $8(1), \quad 1$. https://doi.org/10.1186/s40479-020-00142-6

[21] Saunders, K. E., \&amp; Smith, K. A. (2016). Interventions to prevent self-harm: What does the evidence say? Evidence Based Mental Health, 19(3), 69 - 72. https://doi.org/10.1136/eb-2016-102420

[22] Dear, M., \& Laws, G. (1986). Anatomy of a decision: Recent land use zoning appeals and their effect on group home locations in Ontario. Canadian Journal of Community Mental Health,5(1), 5 - 17. https://doi.org/10.7870/cjcmh-1986-0001

[23] Smart, L., \& Wegner, D. M. (1999). Covering up what can't be seen: Concealable stigma and mental control. Journal of Personality and Social $\begin{array}{llll}\text { Psychology, 77(3), } & 474 & - & 486 .\end{array}$ https://doi.org/10.1037/0022-3514.77.3.474

[24] Overton, S. L., \& Medina, S. L. (2008). The stigma of mental illness. Journal of Counseling \& Development, $\quad$ 86(2), 143-151. https://doi.org/10.1002/j.1556-

6678.2008.tb00491.x

[25] Ritsher, J. B., \& Phelan, J. C. (2004). Internalized stigma predicts erosion of morale among psychiatric outpatients. Psychiatry Research, 129(3), 257 - 265. https://doi.org/10.1016/j.psychres.2004.08.003

[26] Bennewith, O. "General Practice Based Intervention to Prevent Repeat Episodes of Deliberate Self Harm:
Cluster Randomised Controlled Trial * Commentary: Clinical Guidelines Have Limitations." BMJ, vol. 324, no. 7348, 2002, pp. 1254

1254.,

https://doi.org/10.1136/bmj.324.7348.1254.

[27] Coleman, Linda Jane, et al. "Getting My Tat on ... and off: Consumer Explanation of Tattoos' Roles in Presentation-of-Self in Everyday Life." Journal of Global Scholars of Marketing Science, vol. 27, no. 1, 2017, $\quad$ pp. 46-59., doi:10.1080/21639159.2016.1265320.

[28] Miller, Brian K., et al. "Body Art in the Workplace: Piercing the Prejudice?" Personnel Review, vol. 38, no. $6, \quad 2009, \quad$ pp. 621-640. doi:10.1108/00483480910992247.

[29] Jibuti, Daviti. "Discrimination against Workers with VISIBLE Tattoos: Experimental Evidence from Germany." SSRN Electronic Journal, 2018, doi:10.2139/ssrn.3283719.

[30] Platt, S., \& Hawton, K. (n.d.). Suicidal behavior and the labour market. The International Handbook of Suicide and Attempted Suicide, 309-384. https://doi.org/10.1002/9780470698976.ch20

[31] Hawton, K., Bergen, H., Waters, K., Ness, J., Cooper, J., Steeg, S., \& Kapur, N. (2012). Epidemiology and nature of self-harm in children and adolescents: Findings from The Multicentre study of self-harm in England. European Child \& Adolescent Psychiatry, 21(7), 369-377. https://doi.org/10.1007/s00787-012-0269-6

[32] Wilkinson, P., Kelvin, R., Roberts, C., Dubicka, B., \& Goodyer, I. (2011). Clinical and psychosocial predictors of suicide attempts and nonsuicidal selfinjury in the Adolescent Depression Antidepressants and psychotherapy trial (adapt). American Journal of Psychiatry, 168(5), 495-501. https://doi.org/10.1176/appi.ajp.2010.10050718

[33] Laukkanen, E., Rissanen, M.-L., Honkalampi, K., Kylmä, J., Tolmunen, T., \& Hintikka, J. (2008). The prevalence of self-cutting and other self-harm among 13- to 18-year-old Finnish adolescents. Social Psychiatry and Psychiatric Epidemiology, 44(1), 23-28. https://doi.org/10.1007/s00127-0080398-x

[34] Ougrin, D., Tranah, T., Stahl, D., Moran, P., \& Asarnow, J. R. (2015). Therapeutic interventions for suicide attempts and self-harm in adolescents: Systematic review and meta-analysis. Journal of the American Academy of Child \& Adolescent Psychiatry,54(2).

https://doi.org/10.1016/j.jaac.2014.10.009 
[35] Hawton, K., Witt, K. G., Salisbury, T. L., Arensman, E., Gunnell, D., Hazell, P., Townsend, E., \& van Heeringen, K. (2016). Psychosocial interventions following self-harm in adults: A systematic review and meta-analysis. The Lancet Psychiatry, 3(8), $740-750$. https://doi.org/10.1016/s22150366(16)30070-0

[36] Morken, I. S., Dahlgren, A., Lunde, I., \& Toven, S. (2019). The effects of interventions preventing selfharm and suicide in children and adolescents: an overview of systematic reviews. F1000Research, 8, 890.

https://doi.org/10.12688/f1000research.19506.2

[37] Turner, B. J., Austin, S. B., \& Chapman, A. L. (2014). Treating Nonsuicidal Self-Injury: A Systematic Review of Psychological and Pharmacological Interventions. The Canadian Journal of Psychiatry, 59(11), 576-585. https://doi.org/10.1177/070674371405901103

[38] Barent Walsh, PhD. (2016). Interventions for SelfHarm: What Works and What Does Not. Worcester, MA. Retrieved September 16, 2021, from https://www.inholland.nl/media/10858/presentatiedr-b-walsh.pdf. 